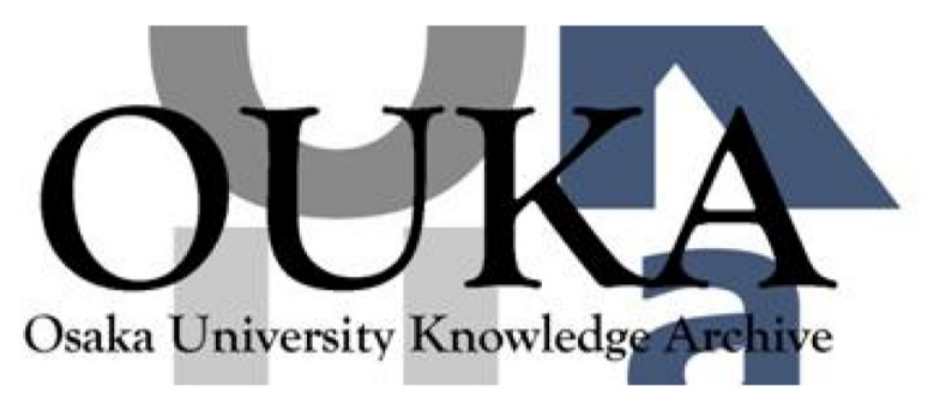

\begin{tabular}{|c|c|}
\hline Title & $\begin{array}{l}\text { Singular Effects of Impurities near the } \\
\text { Ferromagnetic Quantum-Critical Point }\end{array}$ \\
\hline Author(s) & Maebashi, H.; Miyake, K. ; Varma, C. M. \\
\hline Citation & $\begin{array}{l}\text { Physical Review Letters. 88(22) p.226403_1- } \\
\text { p.226403_4 }\end{array}$ \\
\hline Issue Date & $2002-06-03$ \\
\hline oaire:version & VoR \\
\hline URL & ht tps://hdl. hand le. net/11094/2896 \\
\hline rights & $\begin{array}{l}\text { Maebashi, H., Miyake, K., Varma, C. M., } \\
\text { Physical' Review Letters, 88, 22, 226403, 2002- } \\
\text { 06-03. "Copyright } 2002 \text { by the American Physical } \\
\text { Society." }\end{array}$ \\
\hline Note & \\
\hline
\end{tabular}

Osaka University Knowledge Archive : OUKA

https://ir. Library. osaka-u. ac. jp/

0saka University 


\title{
Singular Effects of Impurities near the Ferromagnetic Quantum-Critical Point
}

\author{
H. Maebashi* and K. Miyake \\ Department of Physical Science, Graduate School of Engineering Science, Osaka University, Toyonaka, Osaka 560-8531, Japan
}

\author{
C. M. Varma
}

Bell Laboratories, Lucent Technologies, Murray Hill, New Jersey 07974

(Received 14 September 2001; published 17 May 2002)

\begin{abstract}
Systematic theoretical results for the effects of a dilute concentration of magnetic impurities on the thermodynamic and transport properties in the region around the quantum critical point of a ferromagnetic transition are obtained. In the quasiclassical regime, the dynamical spin fluctuations enhance the Kondo temperature. This energy scale decreases rapidly in the quantum fluctuation regime, where the properties are those of a line of critical points of the multichannel Kondo problem with the number of channels increasing as the critical point is approached, except at unattainably low temperatures where a single channel wins out.
\end{abstract}

DOI: $10.1103 /$ PhysRevLett.88.226403

The asymptotic low temperature singularities in the thermodynamic and transport properties of many solids appear to be due to impurities [1]. While several impurity models have quantum-critical points (QCP), where such singularities may be expected, they require special symmetries unlikely to be present in real systems [2]. An alternate possibility is that the pure system is near a QCP, so that singular low energy fluctuations are present [3-5]. We investigate the possibility in this paper that such fluctuations drive the effects of a dilute concentration of ordinary impurities (not requiring any special symmetries) so that the resulting observable properties are much more singular than those near pure QCP $[6,7]$.

For coupling to nonmagnetic impurities, a general argument on the renormalized impurity scattering was given in relation to insulating behavior in marginal Fermi liquids [8]. It was recently proposed that critical fluctuations enhance potential scattering from nonmagnetic impurities so that the residual resistivity increases as the QCP is approached [9].

Here we investigate the effects of magnetic impurities near a ferromagnetic QCP. The problem is especially interesting for a number of reasons. First, it couples the quantum fluctuation or Kondo effect of magnetic impurities to the singular quantum fluctuations of the pure system. Second, since the transition is at $q=0$ and the order parameter is conserved, conservation laws or Ward identities can be used to obtain systematic results for the effect of impurities just as they are available for the pure ferromagnetic QCP. Third, experimental results on extraordinary pure samples of a ferromagnet-MnSi [10,11], which has a QCP as a function of pressure, are in good accord with the theory [4]. A few results on less pure $\mathrm{MnSi}$ are available [11], which show interesting deviations from that of the purer samples near the QCP. We hope our results will serve as an impetus to further experimental results.

Figure 1 presents a schematic phase diagram around a pure ferromagnetic QCP at $r=0$ where $r$ is a "disorder-
PACS numbers: 71.10.Hf, 72.10.Fk, 75.30.Kz, 75.40.-s

ing" parameter, pressure for MnSi. The crossover between the regimes I and II occurs at $T \propto \xi^{-3}(T)$, where $\xi$ is the magnetic correlation length [3]. In the pure limit, in the quantum-critical regime, the pole in the fluctuation spectra $\chi(\mathbf{q}, \omega)$ has a dispersion $\omega \sim q^{3}$, so that the dynamical critical exponent $z_{\mathrm{d}}=3$. Correspondingly, $\xi(T) \sim T^{-2 / 3}$ in the region I. In the region II, $\xi \sim r^{-1 / 2}$ at low $T$.

The problem of magnetic impurities was looked at long ago by Larkin and Mel'nikov [12]. We have obtained some new results. In the regime I, the net result of enhanced dynamical spin fluctuations at low energies and of a decreased high frequency cutoff is to enhance the Kondo temperature. In the regime II, this enhancement decreases rapidly and an ordinary $S=1 / 2$ magnetic impurity necessarily scatters in many angular momentum channels because of the increasing magnetic correlation length with the number of channels diverging as $r \rightarrow 0$. The crossover from the multichannel to the single-channel behavior occurs at unattainably low temperatures.

We consider the model of $S=1 / 2$ magnetic impurities coupling to the host electrons by the Hamiltonian

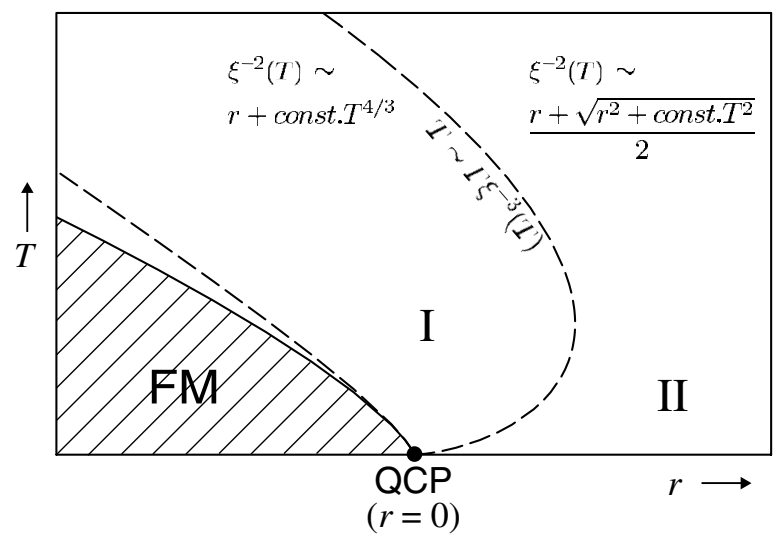

FIG. 1. A schematic phase diagram near a ferromagnetic quantum critical point. 


$$
\frac{J}{2 N} \sum_{\mathbf{k}, \mathbf{k}^{\prime}, \alpha, \beta} \boldsymbol{\sigma}_{\alpha \beta} \cdot \mathbf{S} c_{\mathbf{k}^{\prime} \alpha}^{\dagger} c_{\mathbf{k} \beta} .
$$
pure system is given by

$$
\chi(\mathbf{q}, \omega)=\chi_{0} \kappa_{0}^{2}\left[\kappa^{2}+q^{2}-\mathrm{i} \omega / \Gamma q\right]^{-1},
$$

where $\chi_{0}$ is the magnetic susceptibility of the noninteracting system, $\kappa_{0}$ is of the order of the Fermi wave number $p_{\mathrm{F}}$, and $\kappa \equiv \kappa(T)$ is the inverse of $\xi(T)$ [4]. Hereafter, $r$ is defined by $r \equiv \kappa^{2}(T=0) / 4 p_{\mathrm{F}}^{2}$.

For coupling to magnetic impurities, the vertex renormalization $\Lambda^{(\sigma)}(p, \varpi) / \Lambda_{0}^{(\sigma)}$ is given by a formally exact expression in terms of the irreducible part $\bar{\Lambda}^{(\sigma)}(p, \varpi)$ :

$$
\frac{\Lambda^{(\sigma)}(p, \varpi)}{\Lambda_{0}^{(\sigma)}}=\left(1+\alpha \frac{\chi(\varpi)}{\chi_{0}}\right) \frac{\bar{\Lambda}^{(\sigma)}(p, \varpi)}{\Lambda_{0}^{(\sigma)}},
$$

where $\alpha$ is the coupling constant between the host electrons, $\alpha=2 U \chi_{0}$ for the Hubbard interaction. We have used the simplified notation, $p=(\mathbf{p}, \varepsilon)$ and $\varpi=(\mathbf{q}, \omega)$.

For fluctuations in which $\omega \sim q^{3}$, a version of Migdal theorem holds in the so-called $q$ limit, $\omega \rightarrow 0, q \rightarrow 0$ with
We assume that the dynamical spin susceptibility of the

$\omega / v q=0$ so that the Ward identity requires [13]

$$
\bar{\Lambda}^{(\sigma)} / \Lambda_{0}^{(\sigma)}=\left[z\left(m^{*} / m\right)\left(\alpha+\chi_{0} / \chi\right)\right]^{-1} \simeq 1,
$$

i.e., $z\left(m^{*} / m\right) \simeq \alpha^{-1} \sim 1$ when $\chi_{0} / \chi \ll 1$. By general considerations, $m / m^{*}=z\left(1+\partial \Sigma / \partial \epsilon_{\mathbf{p}}\right)$. So $\partial \Sigma / \partial \epsilon_{\mathbf{p}}$ is not singular while $z^{-1}=1+\partial \Sigma / \partial \varepsilon \sim|\ln (|\varepsilon|, T)|$. Another consequence of Eq. (4) is that the imaginary part of the one-interaction irreducible part of $\chi(\varpi)$ is given by

$\operatorname{Im} \bar{\chi}(\varpi) \simeq \frac{\pi}{2} \sum_{\mathbf{k}}\left[f\left(\varepsilon_{\mathbf{k}^{\prime}}\right)-f\left(\varepsilon_{\mathbf{k}}\right)\right] \delta\left(\omega-\varepsilon_{\mathbf{k}^{\prime}}+\varepsilon_{\mathbf{k}}\right) z^{2}$,

where $\mathbf{k}^{\prime}=\mathbf{k}+\mathbf{q}, \varepsilon_{\mathbf{k}}$ is the energy of the quasiparticles.

The interaction vertex between the host electrons and the pseudofermion field has the form $\Gamma=\Gamma^{(0)}+\Gamma^{(\sigma)} \boldsymbol{\sigma} \cdot \mathbf{S}$. To the leading order in $J$, the vertex is renormalized as

$$
\Gamma^{(\sigma)}\left(p, \omega_{1} ; p+\varpi, \omega_{2}\right)=\frac{J}{2 N} \frac{\Lambda^{(\sigma)}(p, \varpi)}{\Lambda_{0}^{(\sigma)}},
$$

where $\omega_{1}-\omega_{2}=\omega$. Using Eqs. (3) and (4), $\Gamma^{(\sigma)}$ is proportional to $\chi(\varpi)$ in the $q$ limit. In the three-dimensional system with a spherical Fermi surface, it is useful to consider partial-wave components of $\Gamma^{(\sigma)}$ :

$$
\begin{aligned}
\Gamma^{(\sigma)}\left(\mathbf{p}, \varepsilon, \omega_{1} ; \mathbf{p}+\mathbf{q}, \varepsilon+\omega, \omega_{2}\right) & =\frac{J}{2 N} \frac{\alpha \kappa_{0}^{2}}{\kappa^{2}+q^{2}-\mathrm{i} \omega / \Gamma q} \\
& =\sum_{l=0}^{\infty} \frac{2 l+1}{2} \Gamma_{l}^{(\sigma)}\left(\varepsilon, \omega_{1} ; \varepsilon+\omega, \omega_{2}\right) P_{l}(\cos \theta),
\end{aligned}
$$

where $\mathbf{q}^{2}=\left(\mathbf{p}-\mathbf{p}^{\prime}\right)^{2}=p^{2}+p^{\prime 2}-2 p p^{\prime} \cos \theta, P_{l}$ are Legendre functions of the first kind. The real part of $\Gamma_{l}^{(\sigma)}(\omega) \equiv \Gamma_{l}^{(\sigma)}\left(\varepsilon, \omega_{1} ; \varepsilon+\omega, \omega_{2}\right)$ has a singular contribution, while the imaginary part of $\Gamma_{l}^{(\sigma)}(\omega)$ vanishes when $\omega \rightarrow 0$. By use of three roots, $x_{1}, x_{2}$, and $x_{3}$, of a cubic equation, $x^{3}-2 x^{2}+x-\left(\omega / \Gamma \kappa^{3}\right)^{2}=0$, we obtain

$$
\begin{aligned}
\operatorname{Re}_{l}^{(\sigma)}(\omega)=\frac{J}{2 N} \frac{\alpha \kappa_{0}^{2}}{p p^{\prime}} & {\left[\frac{x_{1}\left(1-x_{1}\right)}{\left(x_{3}-x_{1}\right)\left(x_{1}-x_{2}\right)} Q_{l}\left(\zeta_{1}\right)\right.} \\
& +2 \text { cyclic permutations }],
\end{aligned}
$$

where $\zeta_{i}=\left(p^{2}+p^{\prime 2}+\kappa^{2} x_{i}\right) / 2 p p^{\prime}$ for $i=1,2,3$ and $Q_{l}$ are Legendre functions of the second kind. Noting that $\mathcal{Q}_{l}(\zeta)$ have branch points at $\zeta= \pm 1$, we can evaluate the singular contribution in $\Gamma_{l}^{(\sigma)}$ as

$$
\Gamma_{l}^{(\sigma)}(\omega) \simeq \frac{J \alpha}{6 N} \frac{\kappa_{0}^{2}}{p_{\mathrm{F}}^{2}} \ln \frac{D_{l}}{\max \left[|\omega|, \Gamma \kappa^{3}, \Gamma\left|p-p^{\prime}\right|^{3}\right]},
$$

where $\quad D_{l} \equiv \Gamma\left[2 p_{\mathrm{F}} \exp \left(-\sum_{n=1}^{l} n^{-1}\right)\right]^{3} \quad$ serves $\quad$ as $\quad$ an upper cutoff of $|\omega|, \Gamma \kappa^{3}$, or $\Gamma\left|p-p^{\prime}\right|^{3}$ for each $l$. For the Kondo problem we will investigate below, the on-shell scattering $\varepsilon_{\mathbf{p}^{\prime}}=\varepsilon_{\mathbf{p}}+\omega$ is important so that $|\omega|>\Gamma\left|p-p^{\prime}\right|^{3}$. Hence, we put $p=p^{\prime}=p_{\mathrm{F}}$.

If $|\omega|$ is regarded as $\sim T$, Eq. (10) leads us to the following facts: In the regime II, the $\omega$ dependence of $\Gamma_{l}^{(\sigma)}$ is negligible and the number of effective angular momentum channels increases as $l_{\max } \sim 2 p_{\mathrm{F}} \xi$ because the effective size of the impurity scales up as $\xi$ [12]; in the regime I, the $\omega$ dependence of $\Gamma_{l}^{(\sigma)}$ is singular as $\Gamma_{l}^{(\sigma)} \propto \ln \left(D_{l} /|\omega|\right)$ berange not only in space but also in time.

In order to demonstrate carefully how the singular $\omega$ dependence is combined with perturbative renormalization group ( $R G)$ equations for the Kondo problem near the QCP, first we consider the renormalization factor of the pseudofermion propagator $Z(\omega)$. The leading order correction, $\delta Z(\omega)$, with respect to $J$ is given by

$$
\delta Z(\omega)=-\frac{J^{2}}{N^{2}} S(S+1) \sum_{\mathbf{q}} \int_{-\infty}^{0} \frac{d \omega^{\prime}}{\pi} \frac{\operatorname{Im} \chi\left(\mathbf{q}, \omega^{\prime}\right)}{\left(\omega+\omega^{\prime}\right)^{2}} .
$$

Noting that the imaginary part of $\chi$ is given exactly by

$$
\operatorname{Im} \chi(\varpi)=\left|\frac{\Lambda^{(\sigma)}(p, \varpi)}{\bar{\Lambda}^{(\sigma)}(p, \varpi)}\right|^{2} \operatorname{Im} \bar{\chi}(\varpi),
$$

and using Eqs. (4)-(6), Eq. (11) can be evaluated as

$$
\sim S(S+1) \sum_{l=0}^{\infty} \frac{2 l+1}{2} \int_{0}^{D} d \varepsilon \frac{\left|z \Gamma_{l}^{(\sigma)}(\varepsilon)\right|^{2}}{\omega-\varepsilon} \frac{m^{* 2}}{m^{2}} \varrho^{2},
$$

where $\varrho=N m p_{\mathrm{F}} / 2 \pi^{2}$.

From Eq. (13), the variation of $Z\left(\omega_{1}\right)$ on reducing the bandwidth cutoff from $D$ to $D-\delta D$ is obtained cause the effective exchange interaction acts over the long 
immediately. For $\Gamma_{l}^{(\sigma)}\left(\varepsilon_{\mathbf{p}}, \omega_{1} ; \varepsilon_{\mathbf{p}^{\prime}}, \omega_{2}\right)$, the $O\left(J^{2}\right)$ term is

$$
-\frac{z}{2} \frac{m^{*}}{m} \varrho\left[\Gamma_{l}^{(\sigma)}\left(D-\varepsilon_{\mathbf{p}}\right) \Gamma_{l}^{(\sigma)}\left(\varepsilon_{\mathbf{p}^{\prime}}-D\right) \frac{\delta D}{\omega_{1}-D+\varepsilon_{\mathbf{p}}}+\Gamma_{l}^{(\sigma)}\left(-D-\varepsilon_{\mathbf{p}}\right) \Gamma_{l}^{(\sigma)}\left(\varepsilon_{\mathbf{p}^{\prime}}+D\right) \frac{\delta D}{\omega_{1}-D-\varepsilon_{\mathbf{p}^{\prime}}}\right] .
$$

The $O\left(J^{3}\right)$ term of $\Gamma_{l}^{(\sigma)}$ can be evaluated in a similar way to $\delta Z(\omega)$. In Eqs. (13) and (14), $z$ and $m^{*}$ should be understood as functions of the energy scale $D$, respectively. The high-energy spectrum around the band edge of the host electrons may be approximated to be that of the free electrons so that $z \sim 1$ and $m^{*} \sim m$ at an early stage of the RG procedure. Even at low energy, where $z$ vanishes on a logarithmic scale, the product $z\left(\mathrm{~m}^{*} / \mathrm{m}\right)$ remains of the order of 1 by virtue of Eq. (4). Therefore the renormalization of the wave function and of the mass of the host electron does not affect the resulting scaling equation. Neglecting $\varepsilon_{\mathbf{p}} / D, \varepsilon_{\mathbf{p}^{\prime}} / D$, and $\omega_{1} / D$ compared to 1 while keeping the explicit dependence on $\omega=\varepsilon_{\mathbf{p}^{\prime}}-\varepsilon_{\mathbf{p}}$, we obtain the twoloop scaling equation for the invariant coupling $\lambda_{l}(\omega)$ as

$$
\frac{d \lambda_{l}(\omega)}{d \ln D}=-\left|\lambda_{l}(D)\right|^{2}+\lambda_{l}(\omega) \sum_{l^{\prime}=0}^{\infty} \frac{2 l^{\prime}+1}{2}\left|\lambda_{l^{\prime}}(D)\right|^{2} .
$$

Using Eq. (10), the initial bandwidth cutoff in Eq. (15) can be put as $D_{0}=\Gamma\left(2 p_{\mathrm{F}}\right)^{3}$; the bare coupling constant, i.e., the value of $\lambda_{l}(\omega)$ at $D=D_{0}$, is given by $\lambda_{l}^{(\mathrm{b})}(\omega)=$ $(\varrho / \alpha) \Gamma_{l}^{(\sigma)}(\omega)$ and the scaling stops at $D=T$.

The solution of Eq. (15) has the form $\lambda_{l}(\omega)=$ $\lambda_{l}^{(\mathrm{b})}(\omega) A+(J \varrho / N) B_{l}$, where $A$ and $B_{l}$ are functions of $D$ which are independent of $\omega$. Correspondingly, the form of the effective time-dependent interaction in the momentum space divided by $J \varrho / N$, which is given by

$$
\begin{aligned}
A \delta_{0} \mathrm{e}^{-\Omega_{\theta}\left|t-t^{\prime}\right|} \sin \frac{\theta}{2} & +\sum_{l=0}^{l_{\max }}(2 l+1) B_{l} \frac{\delta\left(t-t^{\prime}\right)}{D} \\
& \times P_{l}(\cos \theta),
\end{aligned}
$$

is invariant under the present $\mathrm{RG}$ transformation with scaling time as $t \sim 1 / D$, where $\delta_{0} \equiv \kappa_{0}^{2} / 4 p_{\mathrm{F}}^{2}$ and $\Omega_{\theta} \equiv$ $D \sin (\theta / 2)\left[\sin ^{2}(\theta / 2)+\delta\right]$ with $\delta \equiv \kappa^{2} / 4 p_{\text {F. }}^{2}$. The RG flow of $A$ and $B_{l}$ describes correlation not only between angular momentum channels but also between long-time and instantaneous components of the effective interaction.

If we consider the scaling equation at the one-loop level where the third-order term of Eq. (15) is neglected, we can make a rough estimation of the Kondo temperature $T_{\mathrm{K}}$, which is associated with the breakdown of the perturbation theory, near the QCP. By use of Eq. (10) for $\lambda_{l}^{(b)}(\omega)$, the one-loop scaling equation can be solved analytically. The result depends on whether $\Gamma \kappa^{3}(T)$ is smaller than $T$ or not in accordance with the region I or II as

$$
\lambda_{l}(0)= \begin{cases}\tilde{J} \ln \left(T / \Gamma \kappa^{3}\right)+\sqrt{\tilde{J}} \tan \left[\sqrt{\tilde{J}} \ln \left(D_{l} / T\right)\right] & \mathrm{I} \\ \frac{\sqrt{\tilde{J}} \tan \left[\sqrt{\tilde{J}} \ln \left(D_{l} / \Gamma \kappa^{3}\right)\right]}{1-\sqrt{\tilde{J}} \tan \left[\sqrt{\tilde{J}} \ln \left(D_{l} / \Gamma \kappa^{3}\right)\right] \ln \left(\Gamma \kappa^{3} / T\right)} & \mathrm{II},\end{cases}
$$

where $\tilde{J}=(J \varrho / 6 N)\left(\kappa_{0}^{2} / p_{\mathrm{F}}^{2}\right)$. Since $\lambda_{0}(0) \geq \lambda_{l}(0)$ for arbitrary $l$, we evaluate $T_{\mathrm{K}}$ as the temperature at which $\lambda_{0}(0)$ diverges. Noting $\Gamma \kappa^{3} \simeq D_{0} r^{3 / 2}$ in the region II, we obtain

$$
\frac{T_{\mathrm{K}}}{D_{0}}= \begin{cases}\exp (-\pi / 2 \sqrt{\widetilde{J}}) & r<r^{*} \\ r^{3 / 2} \exp \left(-1 / \sqrt{\tilde{J}} \tan \left[\sqrt{\widetilde{J}}\left|\ln r^{3 / 2}\right|\right]\right) & r>r^{*},\end{cases}
$$

where $r^{*} \equiv \exp (-\pi / 3 \sqrt{\widetilde{J}})$. As a direct consequence of the coupling between the quantum fluctuation of an individual magnetic impurity and dynamical spin fluctuations of host electrons, $T_{\mathrm{K}}$ is seen to be enhanced in the region around a ferromagnetic QCP.

On the basis of the two-loop expansion, the quasiparticle's damping rate $\tau_{\text {imp }}^{-1}(r, T)=z \operatorname{Im} \Sigma_{\text {imp }}(r, T)$ and the electrical resistivity $\rho_{\text {imp }}(r, T)$ due to magnetic impurities can be expressed in terms of $\lambda_{l}(\omega)$ as follows:

$$
\begin{gathered}
\tau_{\text {imp }}^{-1}=\frac{\pi^{2}}{4} S(S+1) \sum_{l=0}^{\infty}(l+1)^{2}\left[\lambda_{l}^{2}(0)-\lambda_{l+1}^{2}(0)\right], \\
\rho_{\text {imp }}=\frac{\pi^{2}}{4} S(S+1) \sum_{l=0}^{\infty}(l+1)\left[\lambda_{l}(0)-\lambda_{l+1}(0)\right]^{2} .
\end{gathered}
$$

By solving Eq. (15) numerically by use of Eq. (9) for $\lambda_{l}^{(\mathrm{b})}(\omega), T$ dependences of $\tau_{\text {imp }}^{-1}$ and $\rho_{\text {imp }}$ are obtained in Fig. 2 , for $\tilde{J}=0.02$ in the weak coupling regime and for several values of $r$. We also show the result of the Born approximation where $\tau_{\text {imp }}^{-1} \propto \xi^{2}(T)$ and $\rho_{\text {imp }} \propto \ln \xi(T)$, which visualizes the crossover line from the regime I to the regime II. The fixed point is that of the single-channel Kondo problem; the multichannel effects can be seen as a transient phenomenon. Note that $T_{\mathrm{K}}$, at which $\rho_{\text {imp }}$ is of the order of 1 , increases rapidly in the region II from $r \sim 0.1$ to $\sim 0.01$ while showing a tendency to be saturated in the region I, consistently with the one-loop result.

In the regime II far away from the QCP, the $\omega$ dependence of the vertex $\Gamma_{l}^{(\sigma)}$ is suppressed, so that the low-energy effective Hamiltonian may be mapped into the anisotropic $n \approx\left(l_{\max }+1\right)^{2}$-channel Kondo model with the bandwidth cutoff $D \approx D_{0} r^{3 / 2}$, in which the coupling constant $\lambda_{l}$ is given by $\lambda_{l}^{(\mathrm{b})}(0)$. By general considerations of the anisotropic multichannel Kondo model, while one enters the influence of the multichannel fixed point for $T$ below $O\left(T_{\mathrm{K}}\right)$, channel anisotropy introduces another energy scale $T_{\mathrm{x}}$ below which the stable single-channel fixed point is reached [14]. Hence, the crossover from the multichannel to the single-channel behavior is predicted when $T_{\mathrm{x}} / T_{\mathrm{K}} \ll 1$. For the $n$-channel case, $T_{\mathrm{K}} \sim D \bar{\lambda}^{n / 2} \exp (-1 / \bar{\lambda})$ and $T_{\mathrm{x}}$ asymptotically scales as $(\Delta \lambda)^{1+n / 2}$, where $\bar{\lambda}$ and $\Delta \lambda$ are the average and difference of coupling constants, respectively [14]. For the two-channel case, a recent numerical RG study has shown that $T_{\mathrm{x}} \simeq D(\Delta \lambda / \bar{\lambda})^{2} \exp (-1 / \bar{\lambda})[15]$. From these results, 


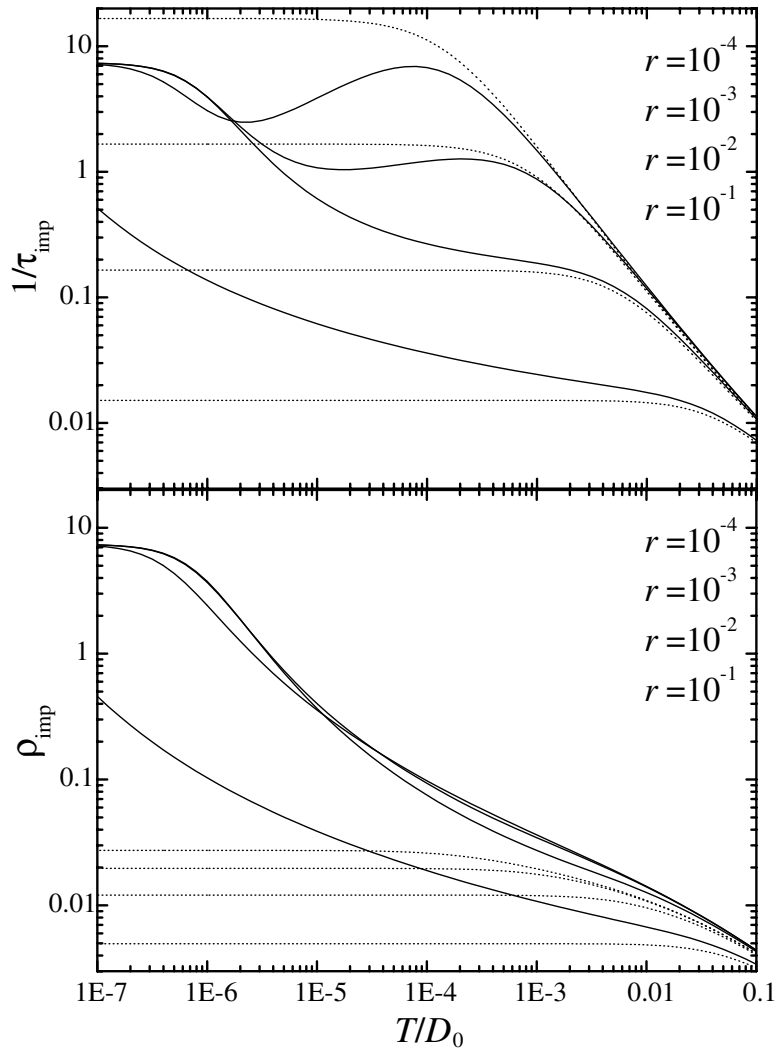

FIG. 2. $T$ dependences of $\tau_{\text {imp }}^{-1}$ and $\rho_{\text {imp }}$ for $\tilde{J}=0.02$, at different $r$ 's $\left(r=10^{-4}, 10^{-3}, 10^{-2}\right.$, and $10^{-1}$ going down, starting from the top curves at high $T$ ), on the basis of the two-loop expansion (solid lines) and the Born approximation (dotted lines).

we expect that $T_{\mathrm{x}}$ is given by $D(\Delta \lambda / \bar{\lambda})^{1+n / 2} \exp (-1 / \bar{\lambda})$, so that $T_{\mathrm{x}} / T_{\mathrm{K}}$ can be evaluated as $(\Delta \lambda)^{1+n / 2} / \bar{\lambda}^{1+n}$. For the present effective Hamiltonian, from Eq. (10), $\lambda_{l} \simeq 3 \tilde{J}|\ln \sqrt{r}|$ while $\lambda_{l}-\lambda_{l+1} \simeq 3 \tilde{J} /(l+1)$. Since $l_{\max } \approx r^{-1 / 2}$, we may make a rough estimation of $T_{\mathrm{x}} / T_{\mathrm{K}}$ as

$$
\frac{T_{\mathrm{x}}}{T_{\mathrm{K}}} \sim(3 \tilde{J})^{-n / 2}|\ln \sqrt{r}|^{-n-1} \approx(\sqrt{3 \tilde{J}}|\ln \sqrt{r}|)^{-1 / r} .
$$

At $r=0.1$ this is $O\left(10^{-3}\right)$ and at $r=0.05$ of $O\left(10^{-8}\right)$ for $\tilde{J}=1$. Therefore there is a possibility of $T_{\mathrm{x}} \ll T_{\mathrm{K}}$ in the intermediate coupling regime. Then for the region of interest away from the QCP in the region II, at experimentally attainable temperatures the observable properties are expected to be those for a line of critical points with singular properties with exponents continuously changing as $r$ changes.

The true fixed point at $r \geq 0$ would be the fixed point of the single-channel Kondo problem. It has zero ground state entropy. For finite $r$ and $T \gg T_{\mathrm{x}}$ in the intermediate coupling regime, we may read off the results for the entropy and its leading temperature dependence from the exact solution for the multichannel problem [16].
On the experimental side, systematic results for the resistivity as a function of $r$ are not available. Further work is required to test the predictions made above.

The authors acknowledge H. Kohno, O. Narikiyo, T. Senthil, and Qimiao Si for clarifying discussions. They also thank I. Affleck, D. L. Cox, H. Kusunose, and $\mathrm{S}$. Yotsuhashi for useful comments on the multichannel Kondo problem. H. M. was supported by the Japan Society for the Promotion of Science for Young Scientists. He also thanks Bell laboratories and Lorentz Center for hospitality during his visits. This work is partly supported by a Grant-in-Aid for COE research (10CE2004) by the Ministry of Education, Science, Sports and Culture.

*Present address: Department of Condensed Matter Physics, The Institute of Scientific and Industrial Research, Osaka University, Ibaraki, Osaka 567-0047, Japan.

[1] For a review, see C. M. Varma, Z. Nussinov, and W. van Saarloos, Phys. Rep. 361, 267 (2002).

[2] P. Nozières and A. Blandin, J. Phys. (Paris) 41, 193 (1980); B. A. Jones, C. M. Varma, and J. W. Wilkins, Phys. Rev. Lett. 61, 125 (1988); O. Sakai and Y. Shimizu, J. Phys. Soc. Jpn. 61, 2333 (1992); I. E. Perakis, C. M. Varma, and A. E. Ruckenstein, Phys. Rev. Lett. 70, 3467 (1993); T. Giamarchi, C.M. Varma, A.E. Ruckenstein, and P. Nozières, Phys. Rev. Lett. 70, 3967 (1993).

[3] J. A. Hertz, Phys. Rev. B 14, 1165 (1976).

[4] T. Moriya, Spin Fluctuations in Itinerant Electron Magnetism (Springer-Verlag, Berlin, 1985).

[5] S. G. Mishra and T. V. Ramakrishnan, Phys. Rev. B 18, 2308 (1978).

[6] For classical phase transitions, the effect of impurities was examined in B. I. Halperin and C. M. Varma, Phys. Rev. B 14, 4030 (1976).

[7] For related work, see A. J. Millis, D. Morr, and J. Schmalian, Phys. Rev. Lett. 87, 167202 (2001).

[8] C. M. Varma, Phys. Rev. Lett. 79, 1535 (1997).

[9] K. Miyake, O. Narikiyo, and Y. Onishi, Physica (Amsterdam) 259B-261B, 676 (1999); K. Miyake and O. Narikiyo, J. Phys. Soc. Jpn. 71, 867 (2002); K. Miyake and H. Maebashi, J. Phys. Soc. Jpn. 71, 1007 (2002).

[10] C. Pfleiderer, G. J. McMullan, S.R. Julian, and G. G. Lonzarich, Phys. Rev. B 55, 8330 (1997).

[11] C. Thessieu et al., Solid State Commun. 95, 707 (1995).

[12] A. I. Larkin and V. I. Mel'nikov, Zh. Eksp. Teor. Fiz. 61, 1231 (1971) [Sov. Phys. JETP 34, 656 (1972)].

[13] For example, see P. Nozières, Theory of Interacting Fermi Systems (Addison-Wesley, Massachusetts, 1997).

[14] H. B. Pang and D. L. Cox, Phys. Rev. B 44, 9454 (1991); I. Affleck, A. W. W. Ludwig, H. B. Pang, and D. L. Cox, ibid. 45, 7918 (1992).

[15] S. Yotsuhashi and H. Maebashi, cond-mat/0203506; J. Phys. Soc. Jpn. (to be published).

[16] I. Affleck and A. W. W. Ludwig, Phys. Rev. B 48, 7297 (1993). 